\title{
Stereospecific Ester Activation in Nitrite-Mediated Carbohydrate Epimerization
}

\author{
Hai Dong, Zhichao Pei, and Olof Ramström* \\ Royal Institute of Technology, Department of Chemistry, Teknikringen 30 \\ S-10044 Stockholm, Sweden. \\ ramstrom@kth.se
}

Supporting Information

\section{Contents}

$\begin{array}{ll}\text { General methods } & \text { S2 }\end{array}$

${ }^{1} \mathrm{H}-\mathrm{NMR}$ spectrum of compound $\mathbf{1 3} \quad \mathrm{S} 3$

${ }^{13} \mathrm{C}-\mathrm{NMR}$ spectrum of compound $\mathbf{1 3} \quad \mathrm{S} 4$

${ }^{1} \mathrm{H}-{ }^{1} \mathrm{H}-\mathrm{COSY}$ spectrum of compound $\mathbf{1 3}$ S5 
General Methods. All commercially available starting materials and solvents were of reagent grade and dried prior to use. Chemical reactions were monitored with thin-layer chromatography using precoated silica gel $60(0.25 \mathrm{~mm}$ thickness $)$ plates. Flash column chromatography was performed on silica gel $60(0.040-0.063 \mathrm{~mm})$. Optical rotations were measured at the sodium D line at ambient temperature. ${ }^{1} \mathrm{H}$ and ${ }^{13} \mathrm{C}$ spectra were recorded with a $400 \mathrm{MHz}$ or $500 \mathrm{MHz}$ instrument at $298 \mathrm{~K}$ in $\mathrm{CDCl}_{3}$, using the residual signals from $\mathrm{CHCl}_{3}\left({ }^{1} \mathrm{H}\right.$ : $\delta=7.25 \mathrm{ppm}$; ${ }^{13} \mathrm{C}: \delta=77.2 \mathrm{ppm}$ ) as internal standard. ${ }^{1} \mathrm{H}$ peak assignments were made by first order analysis of the spectra, supported by standard ${ }^{1} \mathrm{H}-{ }^{1} \mathrm{H}$ correlation spectroscopy (COSY). 


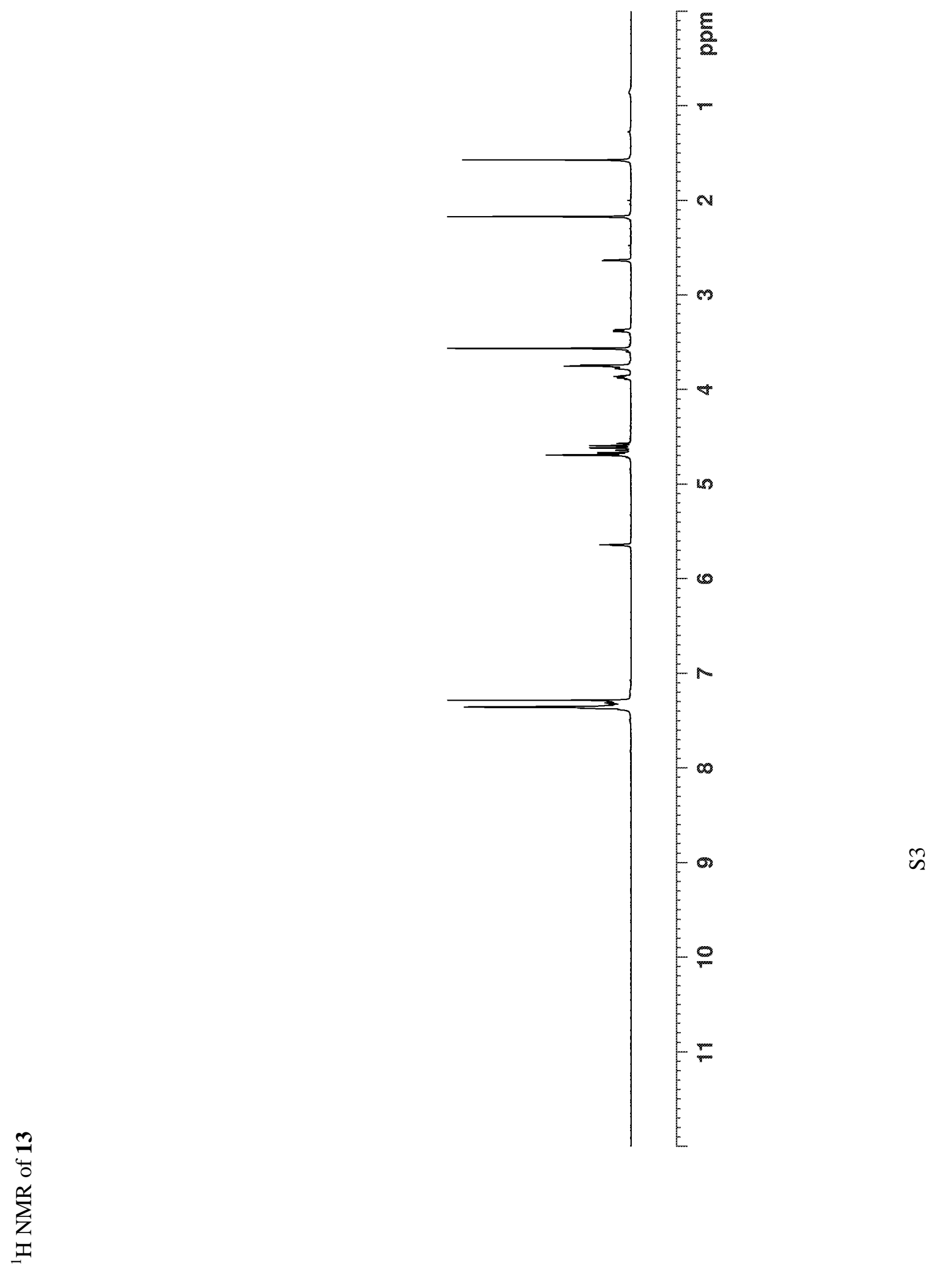




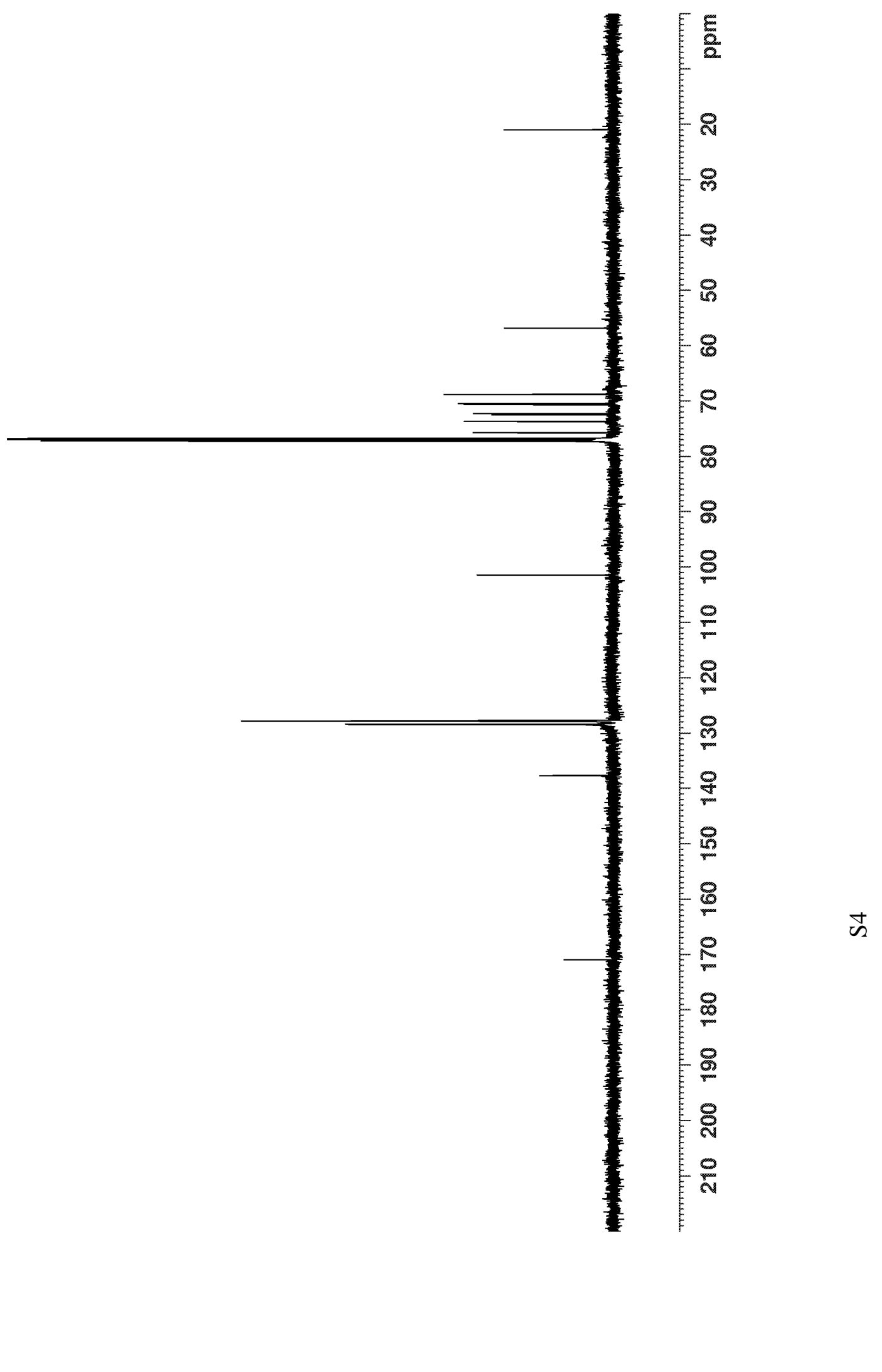




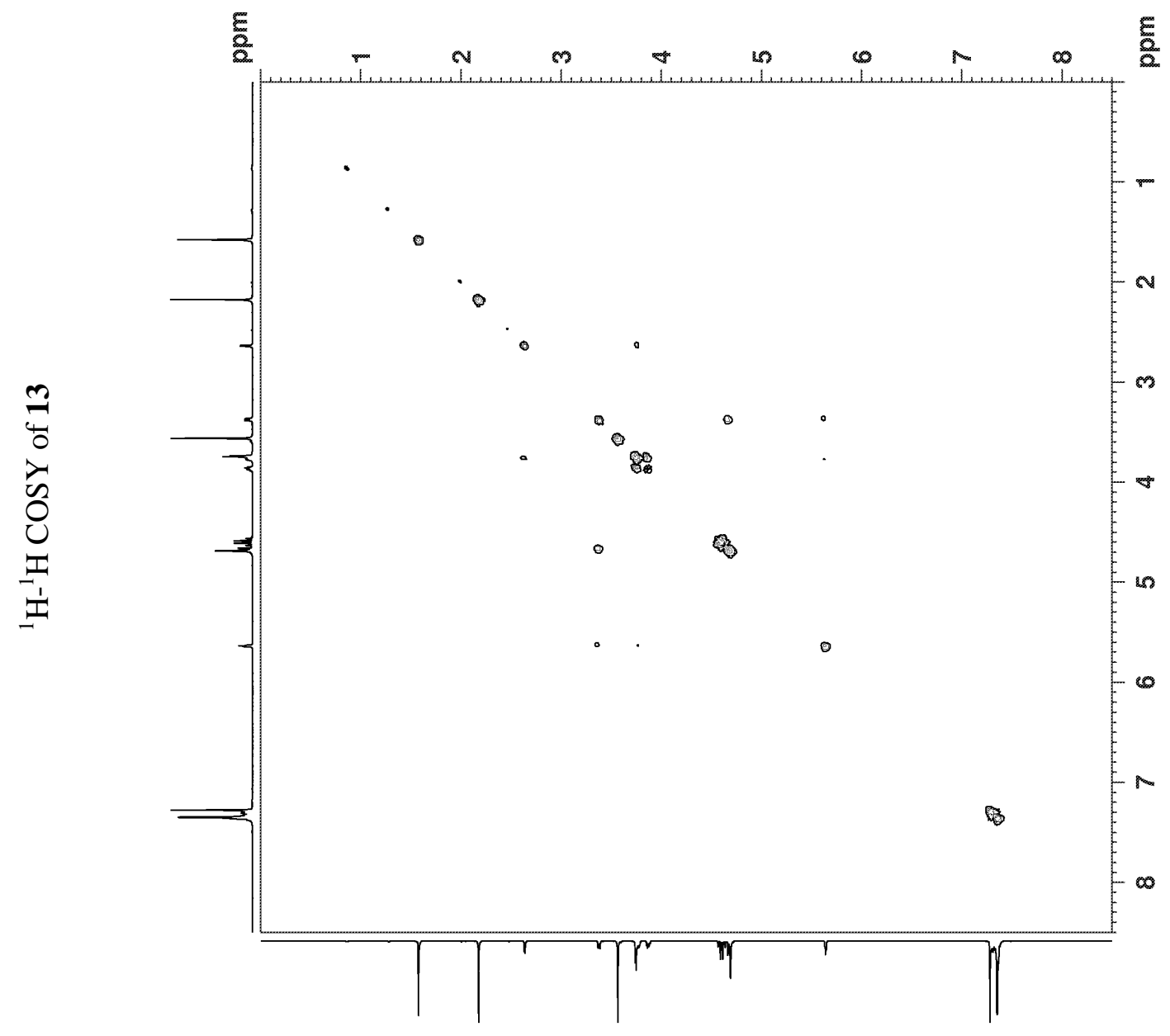

